\title{
Número de folhas para modelar a área foliar de mucuna cinza por dimensões foliares
}

\author{
Number of leaves for modelling the leaf area of velvet bean according to leaf dimensions
}

\author{
Alberto Cargnelutti Filho*, Marcos Toebe, Cláudia Burin, Ismael Mario Marcio Neu \& Bruna Mendonça \\ Alves
}

Universidade Federal de Santa Maria, Santa Maria, RS, Brasil. *Autor para correspondência: alberto.cargnelutti.filho@gmail.com.

Submissão: 23/06/2017 | Aceite: 13/06/2018

\begin{abstract}
RESUMO
Para modelos fidedignos de estimação de área foliar, é importante que as estimativas de seus parâmetros sejam precisas, e, para isso, devem ser geradas com número adequado de folhas. O objetivo deste trabalho foi determinar o número de folhas necessário para modelar a área foliar de mucuna cinza (Stizolobium cinereum) determinada por fotos digitais $(Y)$, em função da largura do limbo do folíolo central da folha $(x)$, por meio do modelo potência $\left(Y=a x^{b}\right)$, gerado por processo iterativo. Em seis períodos de desenvolvimento da cultura $(29,43,57,73,87$ e 101 dias após a emergência) foram coletadas, aleatoriamente, total de 790 folhas. Cada folha é composta pelos folíolos esquerdo, central e direito. Foi mensurada a largura do limbo do folíolo central $(x)$ e determinada a área foliar (soma da área dos folíolos esquerdo, central e direito) por meio do método de fotos digitais ( $\mathrm{Y})$. O número de folhas, necessário para a estimação dos parâmetros $a$ e $b$ do modelo potência e do coeficiente de determinação do modelo $\left(R^{2}\right)$, foi determinado por reamostragem, com reposição. Em mucuna cinza, o modelo potência $\left(\hat{Y}=4,4019 x^{1,8697}, R^{2}=0,9821\right)$ da largura do limbo do folíolo central ( $x$ ) é adequado para estimar a área foliar obtida por fotos digitais $(Y)$. Mensurar 240 folhas é suficiente para construção de modelos precisos do tipo potência, da área foliar de mucuna cinza determinada por fotos digitais ( $\mathrm{Y}$ ) em função da largura do limbo do folíolo central da folha (x).
\end{abstract}

PALAVRAS-CHAVE: Stizolobium cinereum, dimensionamento amostral, modelo potência.

\begin{abstract}
In order to obtain reliable models for estimating the leaf area, it is important that parameter estimates be accurate and, for that, they must be generated based on an appropriate number of leaves. The objective of this research was to determine the number of leaves required to model the leaf area of velvet beans (Stizolobium cinereum) as determined by digital photos $(Y)$ with regard to the width of the central leaflet limb of the leaf $(x)$, using a potency model $\left(Y=a x^{b}\right)$, generated through iterative process. In six periods of culture development $(29,43,57,73,87$ and 101 days after emergence) were collected, randomly, 790 leaves. Each leaf is composed of left, center and right leaflets. The width of the central leaflet $(x)$ was measured and leaf area (the leaf area sum of the left, center and right leaflets) was determined through the method of digital photos (Y). The number of leaves, necessary for estimating parameters $a$ and $b$ of the potency model and the coefficient of determination $\left(R^{2}\right)$, was determined by resampling, with replacement. In the case of the velvet bean, the potency model $\left(\hat{Y}=4.4019 x^{1.8697}, R^{2}=0.9821\right)$ based on the width of the central leaflet $(x)$ is adequate to estimate the leaf area obtained through digital photos $(Y)$. Measuring 240 leaves is sufficient to build accurate potency models of the velvet bean leaf area determined by digital photos $(\mathrm{Y})$ with regard to the width of the central leaflet limb of the leaf $(\mathrm{x})$.
\end{abstract}

KEYWORDS: Stizolobium cinereum, sampling size, potency model.

\section{INTRODUÇÃO}

A cultura de mucuna cinza (Stizolobium cinereum) possui elevada capacidade de fixação biológica de nitrogênio, com cobertura total do solo em até 60 dias após a semeadura (TEODORO et al. 2011). Além disso, ARGENTON et al. (2005) verificaram que a mucuna cinza intercalada com milho, em preparo reduzido do solo, resultou na melhoria das características físicas do solo, com aumento da macroporosidade e da porosidade total e redução da densidade do solo. As espécies de mucuna são capazes de emergir sob 
elevadas quantidades de palha de cana-de-açúcar e em profundidades de até $8 \mathrm{~cm}$, resultando em plantas com altura superior a $96 \mathrm{~cm}$, área foliar maior que $723 \mathrm{~cm}^{2}$ por planta e acúmulo de massa seca superior a 2,09 gramas por planta, até os 35 dias após a semeadura (SILVA et al. 2013).

A área foliar tem relação direta com a interceptação de luminosidade, eficiência fotossintética, taxa de evapotranspiração e respostas aos fertilizantes e à irrigação (BLANCO \& FOLEGATTI 2005) e, portanto, deve ser mensurada de forma acurada. A mensuração da área foliar de forma direta é destrutiva, e pode ser realizada por meio de aparelhos integradores de área foliar, de scanners ou de câmeras digitais (BLANCO \& FOLEGATTI 2005), que são considerados caros e complexos (DEMIRSOY et al. 2005). Alternativamente, a área foliar pode ser estimada indiretamente e de modo não destrutivo, via modelos de estimação de área foliar, gerados com base em dimensões lineares da folha, como comprimento ou largura ou o produto do comprimento $\times$ largura (CARGNELUTTI FILHO et al. 2012a, CARGNELUTTI FILHO et al. 2012b). Modelos precisos de estimação da área foliar de morangueiro (DEMIRSOY et al. 2005), cafeeiro (ANTUNES et al. 2008), pinhão manso (POMPELLI et al. 2012), nabo forrageiro (CARGNELUTTI FILHO et al. 2012b), mucuna cinza (CARGNELUTTI FILHO et al. 2012a) e feijão de porco (TOEBE et al. 2012) têm sido gerados.

Para a estimação da área foliar, o modelo potência, com base no produto comprimento $\times$ largura da folha para as culturas de cafeeiro (ANTUNES et al. 2008), nabo forrageiro (CARGNELUTTI FILHO et al. 2012b) e pinhão manso (POMPELLI et al. 2012) e com base na largura da folha para mucuna cinza (CARGNELUTTI FILHO et al. 2012a) e feijão de porco (TOEBE et al. 2012), foi considerado preciso e adequado. Modelos de estimação de área foliar com elevada precisão, confiabilidade e sem viés devem ser gerados com base em amostra (número de folhas) representativa. Para as culturas de pinhão manso (POMPELLI et al. 2012), de cafeeiro (ANTUNES et al. 2008) e de feijão de porco (CARGNELUTTI FILHO et al. 2015), determinaram que no mínimo 415, 200 e 200 folhas, respectivamente, são suficientes para gerar modelos precisos.

Na cultura de mucuna cinza, o modelo potência $\left(\hat{Y}=3,6450 x^{1,9479}, R^{2}=0,9886\right)$, para estimação da área foliar obtida por fotos digitais $(Y)$ em função da largura do limbo do folíolo central $(x)$, gerado a partir de 650 folhas e validado com 140 folhas, foi considerado apropriado (CARGNELUTTI FILHO et al. 2012a). Porém, os autores não determinaram o tamanho de amostra (número de folhas) adequado para a geração desse modelo. Adicionalmente, o método dos mínimos quadrados foi utilizado para estimar os parâmetros $a$ e $b$ e o coeficiente de determinação $\left(R^{2}\right)$ da função $Y=a x^{b}$ mediante a transformação logarítmica para a linearização da função $Y=a x^{b}$ (STEEL et al. 1997). A geração de modelos do tipo potência, por processo iterativo para modelos não lineares, com minimização da soma de quadrados dos erros, poderia resultar em melhor ajuste dos modelos.

Assim, o objetivo deste trabalho foi determinar o número de folhas necessário para modelar a área foliar de mucuna cinza determinada por fotos digitais $(Y)$ em função da largura do limbo do folíolo central da folha $(\mathrm{x})$, por meio do modelo potência $\left(\mathrm{Y}=a \mathrm{x}^{b}\right)$, gerado por processo iterativo.

\section{MATERIAL E MÉTODOS}

Foi conduzido um ensaio de uniformidade (experimento em branco), de tamanho $17 \mathrm{~m} \times 17 \mathrm{~m}$ (289 $\mathrm{m}^{2}$ ), com a cultura de mucuna cinza (Stizolobium cinereum) na área experimental do Departamento de

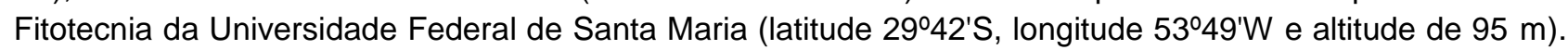
O espaçamento foi de $0,5 \mathrm{~m}$ entre fileiras e $0,125 \mathrm{~m}$ entre plantas na fileira, totalizando 16 plantas $\mathrm{m}^{-2}$. A semeadura foi realizada no dia 13/11/2010 com adubação de base de $40 \mathrm{~kg} \mathrm{ha}^{-1} \mathrm{de} \mathrm{N}, 150 \mathrm{~kg} \mathrm{ha}^{-1} \mathrm{de}_{2} \mathrm{O}_{5}$ e $100 \mathrm{~kg} \mathrm{ha}^{-1}$ de $\mathrm{K}_{2} \mathrm{O}$. A data de emergência foi 22/11/2010, quando $50 \%$ das sementes haviam emergido. Todos os procedimentos culturais (preparação e manejo da área experimental, adubação, semeadura e tratos culturais) foram os mesmos em toda a área experimental, conforme preconizado para esses experimentos de campo, denominados ensaios de uniformidade.

$\mathrm{Na}$ área central do ensaio de uniformidade, com as dimensões de $16 \mathrm{~m} \times 16 \mathrm{~m}\left(256 \mathrm{~m}^{2}\right)$, foram coletadas, aleatoriamente, 90 folhas, aos 29 dias após a emergência (DAE), e 140 folhas em outros cinco períodos $(43,57,73,87$ e $101 \mathrm{DAE})$, totalizando 790 folhas compostas de três folíolos (esquerdo, central e direito). Particularmente, neste estudo, embora as coletas tenham sido realizadas no tempo, foram em distintas plantas e folhas e, assim, foram consideradas como independentes. Em cada planta amostrada, foram coletadas, aleatoriamente, algumas folhas dos terços inferior, médio e superior, a fim de obter amostra representativa do dossel das plantas. Foram coletadas apenas folhas completamente expandidas. Considerou-se folha expandida quando os folíolos da folha do nó superior à folha coletada não mais se tocavam. Essas coletas de um amplo número de folhas (790 folhas), em distintas partes das plantas e em distintos períodos de desenvolvimento foram realizadas com o objetivo de contemplar a variabilidade de 
formatos e dimensões de folhas da cultura, de tal forma que a amostra de folhas fosse representativa para 0 estudo.

Em cada uma das 790 folhas foi mensurada a largura do limbo do folíolo central (x) (Figura 1A) com régua milimetrada. Após, cada folha (composta pelos folíolos esquerdo, central e direito), foi colocada sob vidro transparente, tendo-se o cuidado de mantê-la esticada para evitar sobreposição de área. Em seguida, foi fotografada com câmera digital da marca Sony, modelo DSC-W110, disposta numa base perpendicular a $50 \mathrm{~cm}$ de distância em relação à folha, usando resolução de três megapixels. Essas imagens (fotos) foram processadas com o programa Sigma Scan Pro v.5.0 ${ }^{\circledR}$ (JANDEL SCIENTIFIC 1991), para a determinação da área foliar (soma da área foliar dos folíolos esquerdo, central e direito) por meio do método de fotos digitais $(\mathrm{Y})$.

A

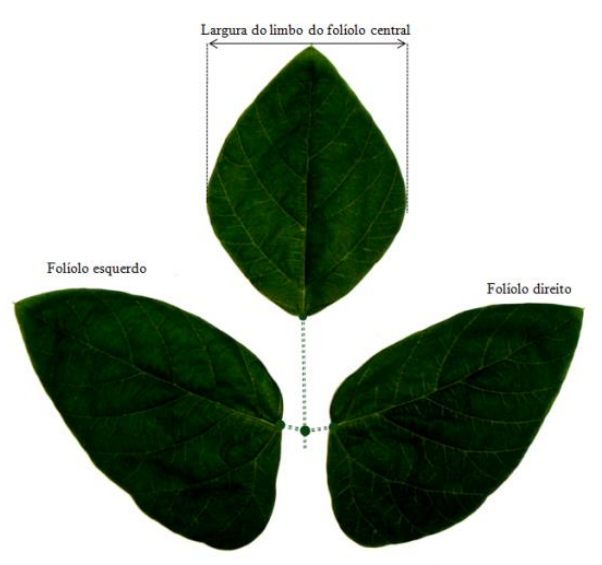

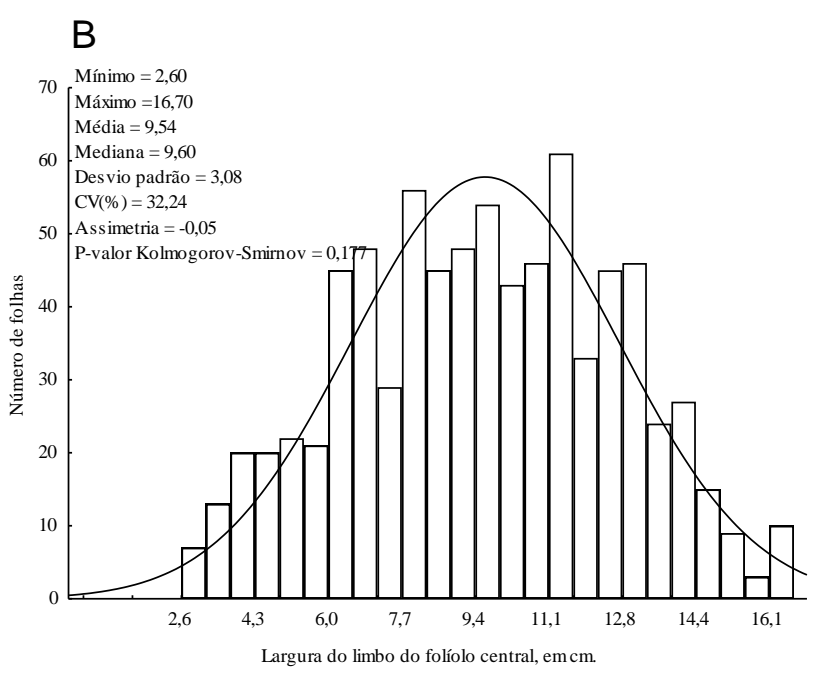
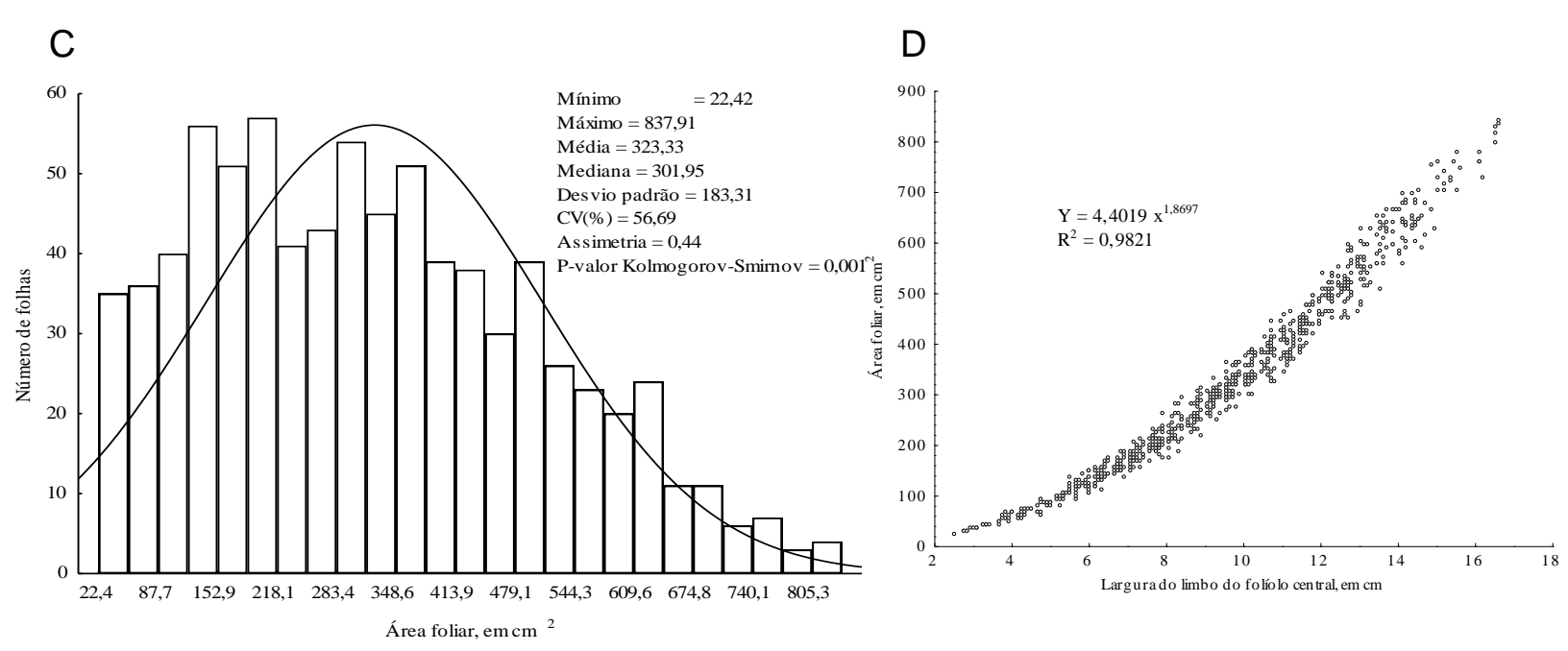

Figura 1. Representação de uma folha de mucuna cinza (Stizolobium cinereum) que é composta pelos folíolos esquerdo, central e direito (A). Histogramas de frequência, estatísticas descritivas e curva de distribuição normal da largura do limbo do folíolo central, em $\mathrm{cm},(B)$ e da área foliar, em $\mathrm{cm}^{2}$ (C) de 790 folhas de mucuna cinza, coletadas em seis períodos de desenvolvimento $(29,43,57$, 73, 87 e 101 dias após a emergência). Diagrama de dispersão e modelo potência da área foliar (Y) em função da largura do limbo do folíolo central $(x)(D)$, gerado com base nas 790 folhas de mucuna cinza, e o coeficiente de determinação $\left(R^{2}\right)$.

Figure 1. Representation of a leaf from velvet bean (Stizolobium cinereum) formed by left, central, and right leaflets $(A)$. Frequency histograms, descriptive statistics and normal distribution curve of the central leaflet width, in $\mathrm{cm}(B)$, and leaf area, in $\mathrm{cm}^{2}(C)$ of 790 velvet bean leaves, collected at six crop development stages $(29,43,57,73,87$, and 101 days after emergence). Scatterplot and power model of the leaf area $(Y)$ as a function of the central leaflet width $(x)(D)$, generated using data from 790 velvet bean leaves, and the coefficient of determination of the model $\left(R^{2}\right)$. 
A partir dos 790 valores de $\mathrm{x}$ e $\mathrm{Y}$, foram calculadas medidas de tendência central e de variabilidade, coeficientes de variação e de assimetria, verificada a normalidade por meio do teste de KolmogorovSmirnov e construídos histogramas de frequência e diagrama de dispersão. Posteriormente, com os dados das 790 folhas, modelou-se a área foliar determinada por fotos digitais $(Y)$ em função da largura do limbo do folíolo central $(\mathrm{x})$, por meio do modelo potência $\left(\mathrm{Y}=a \mathrm{x}^{\mathrm{b}}\right)$, por processo iterativo até a convergência. Estas 790 folhas foram utilizadas por CARGNELUTTI FILHO et al. (2012a). Os autores utilizaram 650 folhas para a geração e 140 folhas para a validação de modelos linear, quadrático e potência. Concluíram que o modelo potência $\left(\mathrm{Y}=a \mathrm{x}^{b}\right)$ foi o mais adequado para estimar a área foliar determinada por fotos digitais $(\mathrm{Y})$ em função da largura do limbo do folíolo central $(x)$. Assim, nesse estudo, com a finalidade de determinar o número de folhas necessário para modelar $Y$ em função de $x$, por meio do modelo potência $\left(Y=a x^{b}\right)$, optou-se por utilizar todas as folhas ( $n=790$ folhas), somente na geração de modelos, para que a amostra tivesse a melhor representatividade possível e, por considerar não ser necessária nova validação.

Nesse estudo, o tamanho de amostra (número de folhas) necessário para o modelo potência $\left(Y=a x^{b}\right)$, foi determinado por reamostragens, com reposição, e foi usado o processo iterativo para estimação dos parâmetros. Para as reamostragens, foram planejados 791 tamanhos de amostra, sendo o tamanho de amostra inicial de 10 folhas (nesse estudo considerado como tamanho mínimo necessário para a construção do modelo), e os demais obtidos com incrementos de uma unidade, até atingir 800 folhas. Portanto, foram planejados os tamanhos de amostra de 10 a 800 folhas.

Após, para cada tamanho de amostra planejado, foram realizadas 3.000 reamostragens, com reposição. Para cada reamostra, foram obtidas as estimativas dos parâmetros $a$ e $b$ do modelo potência $\left(\mathrm{Y}=a \mathrm{x}^{b}\right)$ e o coeficiente de determinação $\left(\mathrm{R}^{2}\right)$. As estimativas de $a, b$ e $\mathrm{R}^{2}$, foram obtidas por processo iterativo, até a convergência, com a finalidade de minimizar a soma de quadrados dos erros. Assim, para cada tamanho de amostra, foram obtidas 3.000 estimativas de $a, b$ e $\mathrm{R}^{2}$ e determinados o percentil 2,5\%, a média e o percentil $97,5 \%$. Depois, calculou-se a amplitude do intervalo de confiança de $95 \%$ (AIC) pela diferença entre o percentil $97,5 \%$ e o percentil $2,5 \%$.

Deve-se interpretar que quanto menor for a AIC mais precisas são as estimativas de $a, b$ e $\mathrm{R}^{2}$. Então, seria possível determinar o número de folhas necessário para atingir valores desejados de AIC para $a, b$ e $\mathrm{R}^{2}$. No entanto, não há valores para $a, b$ e $\mathrm{R}^{2}$ que possam ser tomados como referência. Assim, como critério estatístico, para definição do tamanho de amostra optou-se pelo seguinte: inicialmente, para $a, b$ e $\mathrm{R}^{2}$, a AIC obtida com o menor tamanho de amostra (10 folhas, $\left.A \mathrm{IC}_{10}\right)$ foi considerada como referência, ou seja, como 100\% (máxima AIC e, portanto, mínima precisão nas estimativas de $a, b$ e $R^{2}$ ). A seguir, foi calculado o ganho em precisão $\left(\mathrm{GP}_{\mathrm{i}}\right.$, em \%) com o acréscimo de i-ésimas folhas ( $i=1,2, \ldots, 790$ folhas, respectivamente, para os tamanhos de amostra $11,12, \ldots, 800$ folhas), por meio da expressão: $G P_{i}=100$ $\left(\mathrm{AlC}_{\mathrm{i}} / \mathrm{AlC}_{10}\right) \times 100$, em que: $\mathrm{AIC}$ é a amplitude do intervalo de confiança de $95 \%$ dos tamanhos de amostra 11,12 até 800 folhas. Posteriormente, definiu-se o tamanho de amostra (número de folhas) como sendo aquele em que o ganho em precisão, para $a, b$ e $\mathrm{R}^{2}$, foi no mínimo de $80 \%$.

O percentil $2,5 \%$, a média e o percentil $97,5 \%$ dos tamanhos de amostra de $a, b$ e $\mathrm{R}^{2}$ foram plotados em gráficos, com intervalo de 10 plantas, para melhor representação visual. Apresentou-se, em uma tabela, o ganho em precisão, com intervalo de 10 plantas, para reduzir a dimensionalidade de resultados, e, por considerar, ser suficientemente informativa a apresentação dessa forma. As análises estatísticas foram realizadas com o auxílio do programa $R$ (R DEVELOPMENT CORE TEAM 2017) e do aplicativo Microsoft Office Excel ${ }^{\circledR}$.

\section{RESULTADOS E DISCUSSÃO}

A média da largura do limbo do folíolo central (x) das 790 folhas foi $9,54 \mathrm{~cm}$, com valores mínimos e máximos de, respectivamente, 2,60 e $16,70 \mathrm{~cm}$ (Figura 1B). A área foliar $(Y)$ média foi de $323,33 \mathrm{~cm}^{2}$, oscilando entre 22,42 e $837,91 \mathrm{~cm}^{2}$ (Figura 1C). A ampla variabilidade de $\mathrm{x}$ e $\mathrm{Y}$, ou seja, coeficientes de variação de 32,24 e 56,69\%, respectivamente, é importante para a modelagem. Nesse sentido, CARGNELUTTI FILHO et al. (2012b) e TOEBE et al. (2012) também utilizaram folhas com ampla variabilidade na modelagem de área foliar em nabo forrageiro e feijão de porco, respectivamente. Os autores discutem que a ampla variabilidade dos dados é importante na construção de modelos, pois permite a utilização destes para folhas de diferentes tamanhos e mensuradas em distintos períodos de desenvolvimento da cultura. Embora o experimento tenha sido conduzido em um único ambiente e com único genótipo, a amostragem foi em elevado número de folhas ( $n=790$ folhas), coletadas em todo o dossel das plantas (terços inferior, médio e superior) e em seis períodos de desenvolvimento da cultura, o que possibilitou ampla variabilidade de $\mathrm{x}$ e $\mathrm{Y}$. Com isso, pode-se inferir que esse banco de dados é referência 
para essa cultura, pois contempla folhas de distintas plantas e partes da planta, de distintos estádios de desenvolvimento e, consequentemente, de distintas dimensões e formatos. Adicionalmente, para a largura do limbo do folíolo central (x), com base na proximidade da média com a mediana, na assimetria próxima de zero e no valor-p do teste Kolmogorov-Smirnov, pode-se inferir boa aderência à distribuição normal (Figura 1B). Já $Y$ teve leve afastamento da distribuição normal, caracterizada por média $\left(323,33 \mathrm{~cm}^{2}\right)$ maior que a mediana $\left(301,95 \mathrm{~cm}^{2}\right)$ e assimetria positiva (Figura 1C). Diante desses aspectos, embora verificado leve afastamento de $\mathrm{Y}$ em relação à normalidade, pode-se inferir que esse banco de dados é fidedigno e adequado para a geração de modelos e para o estudo do dimensionamento amostral (número de folhas).

Visualmente, percebe-se no diagrama de dispersão entre $x$ e $Y$ das 790 folhas de mucuna cinza, que há padrão de associação não linear (Figura 1D), o que sugere adequabilidade do modelo potência, conforme já constatado por CARGNELUTTI FILHO et al. (2012a). Modelos do tipo potência, para a estimação da área foliar, também foram ajustados para as culturas de cafeeiro (ANTUNES et al. 2008), nabo forrageiro (CARGNELUTTI FILHO et al. 2012b), pinhão manso (POMPELLI et al. 2012) e feijão de porco (TOEBE et al. 2012). Apesar do uso do mesmo banco de dados, as pequenas diferenças na magnitude das estimativas de $a, b$ e $R^{2}$ do modelo obtido nesse estudo $\left(\hat{Y}=4,4019 x^{1,8697}\right.$ com $R^{2}=0,9821$, com $n=790$ folhas e por processo iterativo, até a convergência, com a finalidade de minimizar a soma de quadrados dos erros), comparado ao determinado em CARGNELUTTI FILHO et al. (2012a) $\left(\hat{Y}=3,6450 x^{1,9479}, R^{2}=0,9886\right.$, com $n=650$ folhas para geração e 140 para validação do modelo, por processo de linearização e mínimos quadrados), podem ser atribuídas ao número de folhas utilizado e o método de estimação dos parâmetros de modelo. Portanto, embora os dois modelos possam ser utilizados, o modelo desse estudo ( $\left.\hat{Y}=4,4019 x^{1,8697}, R^{2}=0,9821\right)$, determinado por processo iterativo e com base em todas as folhas (maior representatividade) deve ser o preferido.

A partir das 3.000 reamostras de 10 folhas (menor tamanho utilizado nesse estudo), a amplitude do intervalo de confiança de $95 \%$ (AIC), foi de 4,4651, 0,4472 e 0,0479 e a média das 3.000 foi 4,2849, 1,8966 e 0,9835 , respectivamente, para as estimativas de $a, b$ e $\mathrm{R}^{2}$ (Figura 2 e Tabela 1). Em outra extremidade, a partir das 3.000 reamostras de 800 folhas (maior tamanho utilizado), a AIC foi de 0,4782, 0,0453 e 0,0046 e a média das 3.000 foi 4,4016, 1,8699 e 0,9821, respectivamente, para as estimativas de $a, b$ e $\mathrm{R}^{2}$ (Figura 2 e Tabela 1). Embora as médias das estimativas de $a, b$ e $\mathrm{R}^{2}$, a partir de 10 e de 800 folhas tenham sido semelhantes, as maiores amplitudes a partir de 10 folhas comparadas as obtidas com 800 folhas, evidenciam que com 10 folhas as estimativas dos parâmetros do modelo tem baixa precisão, podendo resultar em estimativas imprecisas de área foliar e com viés, quando a amostra é insuficiente. Dessa forma, pode-se inferir que modelos gerados a partir de reduzido número de folhas não devem ser utilizados em estudos de estimação de área foliar, e também que é importante e necessário definir o tamanho de amostra adequado para geração de modelos mais precisos.

A amplitude do intervalo de confiança de $95 \%$ (AIC), das estimativas de $a, b$ e $\mathrm{R}^{2}$, diminuiu gradativamente com o acréscimo do número de folhas (Figura 2 e Tabela 1). Esse resultado é esperado, e indica que o aumento do número de folhas proporciona melhoria na precisão das estimativas e, consequentemente, modelos mais confiáveis. Visualmente, percebe-se na Figura 2, que houve decréscimo acentuado da AIC até, aproximadamente 240 folhas. Após os decréscimos são menores, o que indica que o trabalho para a medição de mais folhas resultaria em benefícios inexpressivos na precisão das estimativas dos parâmetros do modelo. Dessa forma, para as estimativas de $a, b$ e $\mathrm{R}^{2}$, pode-se sugerir, visualmente, que 240 folhas seriam suficientes para gerar o modelo potência.

Aumentando o número de folhas, de 10 para 20, houve ganhos em precisão de 27,15\% [100-

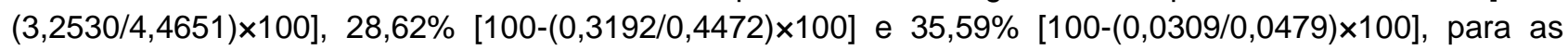
estimativas de $a, b$ e $\mathrm{R}^{2}$, respectivamente. Já ao passar de 10 para 30 folhas, os ganhos em precisão foram $a=40,35 \%, b=41,24 \%$ e $R^{2}=46,16 \%$ (Tabela 1). Percebe-se, portanto, que os ganhos em precisão, com o acréscimo do número de folhas, foram de magnitudes semelhantes para as estimativas de $a$ e $b$ e, relativamente, superior para $R^{2}$. Além disso, os ganhos em precisão foram mais expressivos ao passar de 10 para 20 folhas em relação ao passar de 20 para 30 folhas, e assim, sucessivamente. Ganhos superiores a $80 \%\left(a=80,37 \%, b=81,32 \%\right.$ e $\left.R^{2}=82,26 \%\right)$ foram obtidos ao passar de 10 para 240 folhas. Embora estimativas a partir do maior número de folhas possível devem ser almejadas, a fim de garantir modelos fidedignos, parece razoável estimar esses parâmetros do modelo potência com 240 folhas de mucuna cinza, pois a partir desse número de folhas os ganhos em precisão são inexpressivos. Esse tamanho de amostra ( $n=240$ folhas) é, relativamente, superior ao estabelecido para gerar modelos de área foliar em cafeeiro, por ANTUNES et al. (2008) ( $n=200$ folhas), em feijão de porco, por CARGNELUTTI FILHO et al. (2015) ( $n=200$ folhas) e inferior ao estabelecido para pinhão manso, por POMPELLI et al. (2012) ( $n=415$ folhas). 

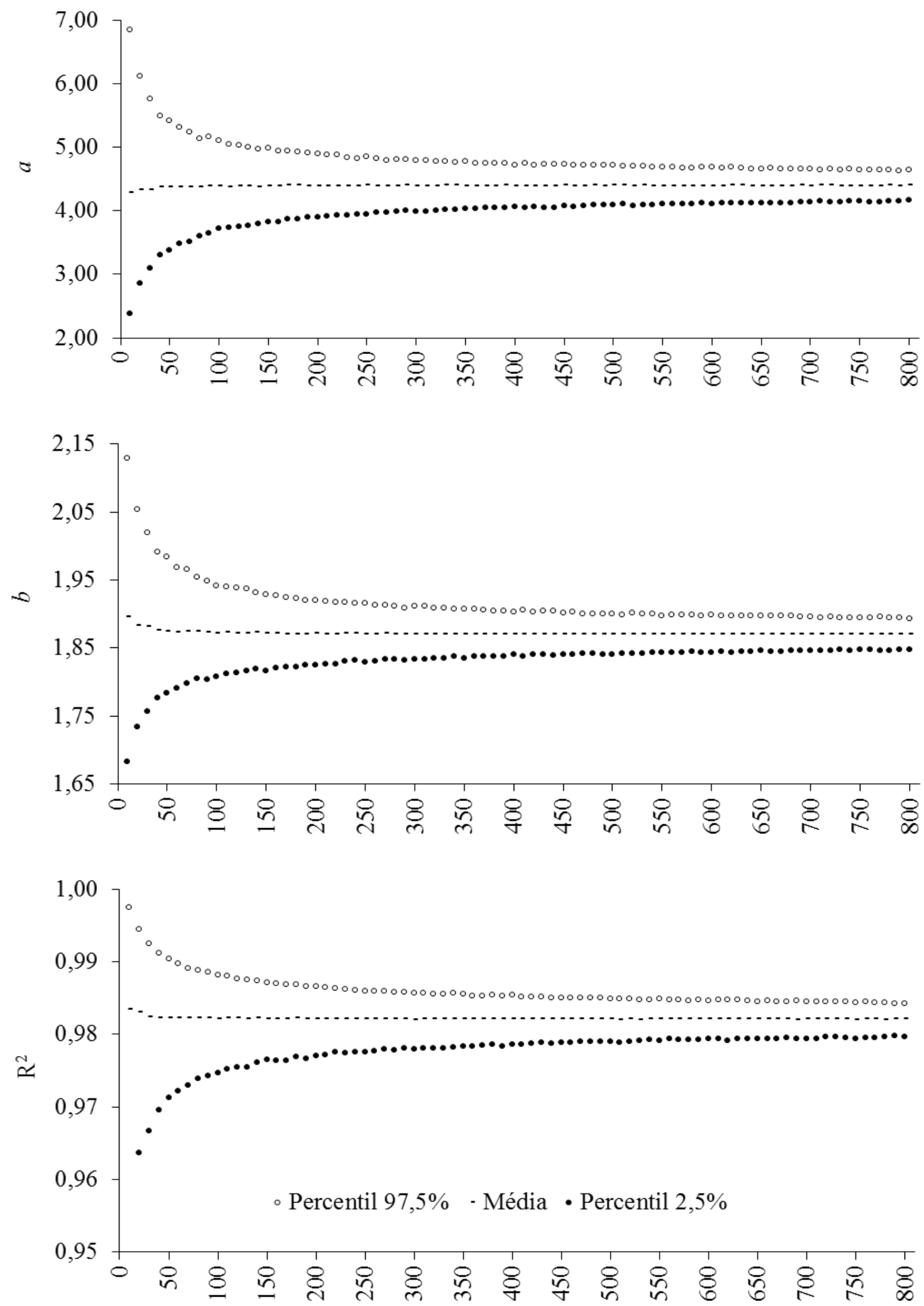

Número de folhas

Figura 2. Percentil $2,5 \%$, média e percentil $97,5 \%$ das 3.000 estimativas dos parâmetros $a$ e $b$ do modelo potência $\left(Y=a x^{b}\right)$, da área foliar de mucuna cinza (Stizolobium cinereum), determinada por fotos digitais $(Y)$ em função da largura do limbo do folíolo central da folha $(x)$ e o coeficiente de determinação $\left(R^{2}\right)$, em cada tamanho de amostra (número de folhas).

Figure 2. Percentile 2.5\%, mean, and percentile $97.5 \%$ for 3,000 estimates of parameter $a$ and $b$ in the power model $\left(Y=a x^{b}\right)$ for the leaf area $(Y)$ of the velvet bean (Stizolobium cinereum) as a function of the central leaflet width $(X)$, generated using a digital photo method for leaf area and the coefficient of determination $\left(R^{2}\right)$. Percentiles and mean are shown for each sample size (number of leaves - batches of ten leaves). 
Tabela 1. Amplitudes dos intervalos de confiança de $95 \%\left(\mathrm{AIC}_{\mathrm{i}}\right)$ e ganhos em precisão $\left(\mathrm{GP}_{\mathrm{i}}\right.$, em \%) das estimativas dos parâmetros $a$ e $b$ do modelo potência $\left(Y=a x^{b}\right)$ e coeficiente de determinação $\left(R^{2}\right)$, que relaciona área foliar de mucuna cinza (Stizolobium cinereum), determinada por fotos digitais $(Y)$, em função da largura do limbo do folíolo central da folha $(x)$, com tamanhos de amostra de 10 , $20, \ldots, 800$ folhas.

Table 1. Values for $95 \%$ confidence interval $\left(A C l_{i}\right)$ and accuracy gain $\left(A G_{i}, \%\right)$ of the estimates of parameters $a$ and $b$ in the power model $\left(Y=a x^{b}\right)$ of leaf area for the velvet bean (Stizolobium cinereum). Leaf area was determined using digital photos, and the model represents leaf area as a function of central leaflet width $(x)$. The coefficient of determination $\left(R^{2}\right)$ for sample sizes $10,20, \ldots$, 800 leaves is also shown.

\begin{tabular}{|c|c|c|c|c|c|c|c|c|c|c|c|c|c|}
\hline № folhas & $\begin{array}{l}\mathrm{AIC}_{\mathrm{i}} \\
a\end{array}$ & $\begin{array}{l}\mathrm{AIC}_{\mathrm{i}} \\
b\end{array}$ & $\begin{array}{l}\mathrm{AlC}_{\mathrm{i}} \\
\mathrm{R}^{2}\end{array}$ & $\begin{array}{l}\mathrm{GP}_{\mathrm{i}} \\
a\end{array}$ & $\begin{array}{l}\mathrm{GP}_{\mathrm{i}} \\
b\end{array}$ & $\begin{array}{l}G P_{i} \\
\mathrm{R}^{2}\end{array}$ & $\begin{array}{l}\text { № } \\
\text { folhas }\end{array}$ & $\begin{array}{l}\mathrm{AlC}_{\mathrm{i}} \\
a\end{array}$ & $\begin{array}{l}\mathrm{AIC}_{\mathrm{i}} \\
b\end{array}$ & $\begin{array}{l}\mathrm{AlC}_{\mathrm{i}} \\
\mathrm{R}^{2}\end{array}$ & $\begin{array}{l}\mathrm{GP}_{\mathrm{i}} \\
a\end{array}$ & $\begin{array}{l}\mathrm{GP}_{\mathrm{i}} \\
b\end{array}$ & $\begin{array}{l}\mathrm{GP}_{\mathrm{i}} \\
\mathrm{R}^{2}\end{array}$ \\
\hline 10 & 4,4651 & 0,4472 & 0,0479 & - & - & - & 410 & 0,7021 & 0,0678 & 0,0065 & 84,28 & 84,85 & 86,41 \\
\hline 20 & 3,2530 & 0,3192 & 0,0309 & 27,15 & 28,62 & 35,59 & 420 & 0,6572 & 0,0626 & 0,0065 & 85,28 & 85,99 & 86,53 \\
\hline 30 & 2,6634 & 0,2628 & 0,0258 & 40,35 & 41,24 & 46,16 & 430 & 0,6778 & 0,0645 & 0,0063 & 84,82 & 85,58 & 86,76 \\
\hline 40 & 2,1897 & 0,2147 & 0,0216 & 50,96 & 51,98 & 54,82 & 440 & 0,6827 & 0,0648 & 0,0063 & 84,71 & 85,52 & 86,82 \\
\hline 50 & 2,0513 & 0,2009 & 0,0191 & 54,06 & 55,08 & 60,17 & 450 & 0,6497 & 0,0618 & 0,0062 & 85,45 & 86,19 & 87,09 \\
\hline 60 & 1,8310 & 0,1774 & 0,0176 & 58,99 & 60,33 & 63,18 & 460 & 0,6525 & 0,0627 & 0,0061 & 85,39 & 85,99 & 87,19 \\
\hline 70 & 1,7365 & 0,1675 & 0,0162 & 61,11 & 62,55 & 66,21 & 470 & 0,6296 & 0,0595 & 0,0060 & 85,90 & 86,69 & 87,39 \\
\hline 80 & 1,5407 & 0,1491 & 0,0149 & 65,49 & 66,66 & 68,81 & 480 & 0,6218 & 0,0589 & 0,0060 & 86,07 & 86,82 & 87,51 \\
\hline 90 & 1,5145 & 0,1456 & 0,0144 & 66,08 & 67,43 & 70,03 & 490 & 0,6281 & 0,0601 & 0,0061 & 85,93 & 86,57 & 87,20 \\
\hline 100 & 1,3881 & 0,1338 & 0,0134 & 68,91 & 70,09 & 71,93 & 500 & 0,6293 & 0,0595 & 0,0059 & 85,91 & 86,69 & 87,59 \\
\hline 110 & 1,3231 & 0,1276 & 0,0127 & 70,37 & 71,47 & 73,41 & 510 & 0,5980 & 0,0567 & 0,0059 & 86,61 & 87,33 & 87,63 \\
\hline 120 & 1,2711 & 0,1244 & 0,0122 & 71,53 & 72,19 & 74,59 & 520 & 0,6187 & 0,0592 & 0,0059 & 86,14 & 86,76 & 87,73 \\
\hline 130 & 1,2381 & 0,1201 & 0,0120 & 72,27 & 73,15 & 74,89 & 530 & 0,6029 & 0,0580 & 0,0057 & 86,50 & 87,04 & 88,08 \\
\hline 140 & 1,1636 & 0,1127 & 0,0112 & 73,94 & 74,79 & 76,67 & 540 & 0,5949 & 0,0569 & 0,0056 & 86,68 & 87,28 & 88,36 \\
\hline 150 & 1,1636 & 0,1114 & 0,0106 & 73,94 & 75,10 & 77,92 & 550 & 0,5729 & 0,0543 & 0,0058 & 87,17 & 87,85 & 87,86 \\
\hline 160 & 1,1132 & 0,1065 & 0,0105 & 75,07 & 76,20 & 78,02 & 560 & 0,5840 & 0,0557 & 0,0054 & 86,92 & 87,54 & 88,74 \\
\hline 170 & 1,0702 & 0,1026 & 0,0105 & 76,03 & 77,05 & 78,17 & 570 & 0,5756 & 0,0554 & 0,0055 & 87,11 & 87,61 & 88,52 \\
\hline 180 & 1,0558 & 0,1012 & 0,0099 & 76,35 & 77,37 & 79,28 & 580 & 0,5589 & 0,0528 & 0,0053 & 87,48 & 88,19 & 88,85 \\
\hline 190 & 1,0111 & 0,0956 & 0,0099 & 77,36 & 78,63 & 79,34 & 590 & 37 & 0,0538 & 0,0054 & 87,38 & 87,96 & 88,68 \\
\hline 200 & 0,9947 & 0,0959 & 0,0097 & 77,72 & 78,55 & 79,81 & 600 & 0,5741 & 0,0545 & 0,0053 & 87,14 & 87,81 & 88,90 \\
\hline 210 & 0,9685 & 0,0938 & 0,0093 & 78,31 & 79,03 & 80,58 & 610 & 0,5500 & 0,0528 & 0,0054 & 87,68 & 88,20 & 88,66 \\
\hline 220 & 0,9556 & 0,0915 & 0,0088 & 78,60 & 79,54 & 81,65 & 620 & 0,5576 & 0,0532 & 0,0056 & 87,51 & 88,10 & 88,22 \\
\hline 230 & 0,9051 & 0,0873 & 0,0088 & 79,73 & 80,49 & 81,57 & 630 & 0,5435 & 0,0523 & 0,0053 & 87,83 & 88,31 & 88,90 \\
\hline 240 & 0,8765 & 0,0835 & 0,0085 & 80,37 & 81,32 & 82 & 640 & 119 & 0,0520 & 0,0052 & 87,86 & 88,38 & 89,07 \\
\hline 250 & 0,9082 & 0,0866 & 0,0083 & 79,66 & 80,63 & 82,65 & 650 & 0,5360 & 0,0509 & 0,0052 & 88,00 & 88,61 & 89,14 \\
\hline 260 & 0,8494 & 0,0825 & 0,0082 & 80,98 & 81,55 & 82,94 & 660 & 0,5491 & 0,0525 & 0,0052 & 87,70 & 88,26 & 89,12 \\
\hline 270 & 0,8187 & 0,0790 & 0,0081 & 81,66 & 82,34 & 83,12 & 670 & 0,5463 & 0,0526 & 0,0051 & 87,76 & 88,24 & 89,33 \\
\hline 280 & 0,8266 & 0,0787 & 0,0080 & 81,49 & 82,41 & 83,29 & 680 & 0,5353 & 0,0516 & 0,0050 & 88,01 & 88,47 & 89,49 \\
\hline 290 & 0,8032 & 0,0765 & 0,0077 & 82,01 & 82,90 & 83,91 & 690 & 0,5117 & 0,0490 & 0,0051 & 88,54 & 89,05 & 89,25 \\
\hline 300 & 0,8105 & 0,0776 & 0,0078 & 81,85 & 82,64 & 83,78 & 700 & 0,5230 & 0,0495 & 0,0051 & 88,29 & 88,93 & 89,30 \\
\hline 310 & 0,8138 & 0,0784 & 0,0076 & 81,77 & 82,47 & 84,1 & 710 & 0,5037 & 0,0484 & 0,0052 & 88,72 & 89,17 & 89,20 \\
\hline 320 & 0,7786 & 0,0740 & 0,0075 & 82,56 & 83,46 & 84,37 & 720 & 0,5227 & 0,0497 & 0,0049 & 88,29 & 88,89 & 89,77 \\
\hline 330 & 0,7590 & 0,0731 & 0,0075 & 83,00 & 83,66 & 84,39 & 730 & 0,4981 & 0,0478 & 0,0048 & 88,85 & 89,30 & 89,89 \\
\hline 340 & 0,7424 & 0,0702 & 0,0074 & 83,37 & 84,31 & 84,55 & 740 & 0,5074 & 0,0480 & 0,0049 & 88,64 & 89,27 & 89,83 \\
\hline 350 & 0,7464 & 0,0717 & 0,0072 & 83,28 & 83,97 & 84,99 & 750 & 0,4999 & 0,0472 & 0,0050 & 88,80 & 89,45 & 89,48 \\
\hline 360 & 0,7223 & 0,0700 & 0,0069 & 83,82 & 84,34 & 85,49 & 760 & 0,4953 & 0,0476 & 0,0050 & 88,91 & 89,35 & 89,66 \\
\hline 370 & 0,7050 & 0,0672 & 0,0068 & 84,21 & 84,97 & 85,86 & 770 & 0,5052 & 0,0484 & 0,0048 & 88,69 & 89,17 & 89,98 \\
\hline 380 & 0,7049 & 0,0671 & 0,0067 & 84,21 & 85,00 & 86,00 & 780 & 0,5041 & 0,0476 & 0,0047 & 88,71 & 89,36 & 90,24 \\
\hline 390 & 0,6957 & 0,0657 & 0,0069 & 84,42 & 85,30 & 85,54 & 790 & 0,4796 & 0,0459 & 0,0046 & 89,26 & 89,74 & 90,50 \\
\hline 400 & 0,6571 & 0,0626 & 0,0067 & 85,28 & 85,99 & 85,98 & 800 & 0,4782 & 0,0453 & 0,0046 & 89,29 & 89,87 & 90,36 \\
\hline
\end{tabular}

$\mathrm{GP}_{\mathrm{i}}=100-\left(\mathrm{AlC}_{\mathrm{i}} / \mathrm{AlC}_{10}\right) \times 100$, em que: $\mathrm{AlC}_{\mathrm{i}}$ é a amplitude do intervalo de confiança de $95 \%$ dos tamanhos de amostra $\mathrm{i}, \mathrm{i}=10,20, \ldots$, 800 folhas e $\mathrm{AIC}_{10}$ é a amplitude do intervalo de confiança de $95 \%$ do tamanho de amostra referência, ou seja, 10 folhas.

Modelos gerados a partir de amostras pequenas (nesse estudo, menores que 240 folhas) devem ser evitados pela imprecisão das estimativas e, portanto, modelos gerados a partir de amostras maiores (nesse estudo, igual ou maiores que 240 folhas) devem ser incentivados. Porém, a partir de determinado tamanho de amostra (número de folhas) os ganhos são desprezíveis em relação ao custo para mensuração das 
dimensões lineares das folhas e da área foliar. Assim, diante dos resultados desse estudo e das inferências supracitadas, parece razoável aceitar que 240 folhas são suficientes para gerar modelos precisos do tipo potência, da área foliar de mucuna cinza determinada por fotos digitais $(\mathrm{Y})$ em função da largura do limbo do folíolo central da folha $(\mathrm{x})$.

\section{CONCLUSÃO}

Em mucuna cinza, o modelo potência $\left(\hat{Y}=4,4019 x^{1,8697}, R^{2}=0,9821\right)$ da largura do limbo do folíolo central $(\mathrm{x})$ é adequado para estimar a área foliar obtida por fotos digitais $(\mathrm{Y})$.

Mensurar 240 folhas é suficiente para construir modelos precisos do tipo potência, da área foliar de mucuna cinza determinada por fotos digitais $(Y)$ em função da largura do limbo do folíolo central da folha (X).

\section{AGRADECIMENTOS}

Ao Conselho Nacional de Desenvolvimento Científico e Tecnológico e à Coordenação de Aperfeiçoamento de Pessoal de Nível Superior, pelas bolsas concedidas. À Fundação de Amparo à Pesquisa do Estado do Rio Grande do Sul, pelo auxílio financeiro e concessão de bolsa de iniciação científica.

\section{REFERÊNCIAS}

ANTUNES WC et al. 2008. Allometric models for non-destructive leaf area estimation in coffee (Coffea arabica and Coffea canephora). Annals of Applied Biology 153: 33-40.

ARGENTON J et al. 2005. Comportamento de atributos relacionados com a forma da estrutura de Latossolo Vermelho sob sistemas de preparo e plantas de cobertura. Revista Brasileira de Ciência do Solo 29: 425-435.

BLANCO FF \& FOLEGATTI MV. 2005. Estimation of leaf area for greenhouse cucumber by linear measurements under salinity and grafting. Scientia Agricola 62: 305-309.

CARGNELUTTI FILHO A et al. 2012a. Estimação da área foliar de mucuna cinza por meio de método não destrutivo. Ciência Rural 42: 238-242.

CARGNELUTTI FILHO A et al. 2012b. Estimativa da área foliar de nabo forrageiro em função de dimensões foliares. Bragantia 71: 47-51.

CARGNELUTTI FILHO A et al. 2015. Number of leaves needed to model leaf area in jack bean plants using leaf dimensions. Bioscience Journal 31: 1651-1662.

DEMIRSOY $\mathrm{H}$ et al. 2005. Improved model for the non-destructive estimation of strawberry leaf area. Fruits 60: 69-73.

JANDEL SCIENTIFIC. 1991. User's Manual. California. 280p.

POMPELLI MF et al. 2012. Allometric models for non-destructive leaf area estimation of Jatropha curcas. Biomass and Bioenergy 36: 77-85.

R DEVELOPMENT CORE TEAM. 2017. A language and environment for statistical computing. Disponível em: http://www.R-project.org/ Acesso em: 22 Jun. 2017.

SILVA GBF et al. 2013. Superação da profundidade de semeadura e densidades de palha para Mucuna aterrima, Mucuna deeringiana e Mucuna cinerea. Planta Daninha 31: 313-317.

STEEL RGD et al. 1997. Principles and procedures of statistics: a biometrical approach. 3.ed. New York: McGraw-Hill. $666 \mathrm{p}$.

TEODORO RB et al. 2011. Aspectos agronômicos de leguminosas para adubação verde no Cerrado do Alto Vale do Jequitinhonha. Revista Brasileira de Ciência do Solo 35: 635-643.

TOEBE M et al. 2012. Modelos para a estimação da área foliar de feijão de porco por dimensões foliares. Bragantia 71: 37-41. 